\title{
Characterization and quantification of gait deficits within gait phases using fuzzy-granular computing*
}

\author{
Melaku A. Bogale ${ }^{1}$, Huiying Yu², Thompson Sarkodie-Gyan ${ }^{2}$, Amr Abdelgawad $^{3}$ \\ ${ }^{1}$ University of Texas at El Paso, Computational Science Program, El Paso, USA \\ ${ }^{2}$ Department of Electrical and Computer Engineering, University of Texas at El Paso, El Paso, USA \\ ${ }^{3}$ Texas Tech University Health Science Center, El Paso, USA \\ Email: mabogale@miners.utep.edu
}

Received 5 October 2012; revised 7 November 2012; accepted 23 November 2012

\section{ABSTRACT}

People with neurological disorders like Cerebral Palsy (CP) and Multiple Sclerosis (MS) suffer associated functional gait problems. The symptoms and sign of these gait deficits are different between subjects and even within a subject at different stage of the disease. Identifying these gait related abnormalities helps in the treatment planning and rehabilitation process. The current gait assessment process does not provide very specific information within the seven gait phases. The objective of this study is to investigate the possible application of granular computing to quantify gait parameters within the seven gait phases. In this process we applied fuzzy-granular computing on the vertical ground reaction force (VGRF) and surface electromyography (sEMG) data to obtain respective characteristic values for each gait phase. A fuzzy similarity (FS) measure is used to compare patient values with age and sex matched control ablebodied group. We specifically applied and tested this approach on 10 patients (4 Cerebral Palsy and 6 Multiple Sclerosis) to identify possible gait abnormalities. Different FS values for VGRF for right and left leg is observed. The VGRF analysis shows smaller FS values during the swing phase in CP and MS subjects that are evidence of associated stability problem. Similarly, FS values for muscle activates of the four-selected muscle display a broad range of values due to difference between subjects. Degraded FS values for different muscles at different stage of the gait cycle are reported. Smaller FS values are sign of abnormal activity of the respective muscles. This approach provides individual centered and very specific information within the gait phases that can be employed for diagnosis, treatment and rehabilitation process.

${ }^{*}$ Conflict of interest: The authors do not have any financial and personal relationships with other people or organizations that could have inappropriately influence (bias) their work.
Keywords: Fuzzy-Granular Algorithm; Gait Phases; Fuzzy Similarity; Cerebral Palsy; Multiple Sclerosis

\section{INTRODUCTION}

For people with mobility disabilities gait analysis is used to provide diagnosis, evaluation and treatment planning information. The benefit of gait analysis is well established that it has now become a part of routine process in many rehabilitation centers [1]. People with Multiple Sclerosis (MS) may suffer from significant gait impairment even at early stage of the disease [2,3]. Gait analysis has been used to identify associated gait deficit with MS $[2,4]$. Gait variability study in people with MS revealed slower walking speed and more fatigue than control healthy group [5]. In the study [6], the effect of MS on the frequency content of vertical ground reaction force (VGRF) during walking was investigated. Compared with health controls significantly lower frequency content in VGRF and no difference in frequency content in anterior-posterior ground reaction forces [6].

Lee EH et al. [7] emphasized the importance of gait analysis in critical surgical decision-making in children with Cerebral Palsy (CP). In this study [7] surgical decisions on children with $\mathrm{CP}$, based on clinical evaluation and gait analysis was shown to help improve gait quality after surgery compared to decisions solely made on clinical assessment. According to [8] gait analysis has been used to make surgical procedure decisions in patients with CP. There are a growing number of literatures [9-15] related to gait analysis and Cerebral Palsy in diagnosis and treatment planning decisions making process. Wavelet analysis was applied to study surface electromyography (sEMG) signals acquired from lower extremity muscles in children with CP [14]. Probabilistic gait classification in children with CP reported in [15]. Cluster analysis was used for identification of sagittal gait patterns [16]. Principal component analysis was applied to extract gait patterns in children with CP [12]. 
Mark J. et al. [17] employed fuzzy-clustering to classify temporal-distance and kinematic gait parameters for CP children.

The dynamic behavior of gait parameters is cyclic and the "normal" or expected pattern (or values) of these parameters over a gait cycle is well known. Studying dynamic gait parameters over a gait cycle helps identify alteration or deviation from the expected reference pattern or values. Specifically, quantifying kinematic, kinetic and surface electromyography (sEMG) gait parameters over a given gait cycle play a crucial role in recognizing associated neurological related gait deficits. Additional techniques and measurements that enable quantification and representation into the seven gait phases add more reliability and specificity to the analysis process. For example, monitoring of the sEMG activity over a gait cycle or a gait phases gives valuable information for diagnosis and treatment decisions [18].

Most gait study techniques are limited to full gait cycle analysis, which focuses on comparison of reference patterns or values with the respective parameters of neurological impaired subjects. Few studies [19-21] investigated gait parameters by decomposing the full gait cycle into its seven phases. In [19-21] the authors implemented fuzzy-ruled approach to divide a gait cycle into its seven phases and make very specific comparisons and analysis within each gait phase. In these studies averaging gait variables values in each phase was used for quantification and representation. Mean value representation may be a good way and works well for slowly time-varying signals. However, averaging is not a good choice for non-smoothly time-varying signals with typical peaks and valleys. Ground reaction forces and muscle activity signal are examples of such rapidly time-varying signals with characteristic shape and peak amplitudes. We believe that a more accurate representation and quantification could be possible by employing granular representation scheme in each phase. Quantification of gait parameter value in each phase based on data-driven granule representation helps to capture the information in each sub-cycle and preserve the experimental significance and justifiability of the signal. We present a possible application of fuzzy-granular computing to investigate the dynamic behavior of the VGRF and sEMG over the seven gait phases. In this regard, we build an information granule based on fuzzy-triangular membership function to characterize, quantify and represent gait parameters values in each sub-cycle. The proposed technique is tested and used to identify and distinguish gait deficits among MS and CP subjects.

\section{GAIT TERMINOLOGIES}

Michael (2007) [1], defined gait cycle as "the time interval between two successive occurrence of one of the re- petitive events of walking”. We can define the gait cycle using any event in the walking process; the most common way of defining a cycle is to use the instance of "initial contact" of one foot. Accordingly, a gait cycle begins at the instant one-foot strikes/contacts ground, and the instant when the same foot strikes the ground again, marks the end of the gait cycle. Based on the major events happening during a gait cycle [1], a full gait cycle is divided into seven gait phases [1]: Loading response ( $0 \%-10 \%$ of the full cycle), mid stance $(10 \%$ $30 \%)$, terminal stance $(30 \%-50 \%)$, pre-swing $(50 \%$ $60 \%)$, initial swing (60\% - 70\%), mid swing (70\% - 85\%) and terminal swing $(85 \%-100 \%)$ [1].

\section{GRANULAR REPRESENTATION OF GAIT PHASES}

To minimize the effect of individual differences in speed of walking and to allow inter-subject comparisons normalization [22] was performed before granulation. The data were normalized as,

$$
S=\frac{S_{0}-\min \left(S_{0}\right)}{\max \left(S_{0}\right)-\min \left(S_{0}\right)}
$$

where $S_{0}$ is the original time-series data, max is maximum, and min is minimum. Normalized VGRF and Soleus sEMG are shown for one complete gait cycle in Figure 1.

Given data sample points for one complete gait cycle, one can use the percentage of each phase from the complete cycle to divide the samples into seven segments or granules (phases in this case). Next we seek granular representation of those samples that fall within each phase. In this paper, we present fuzzy-triangular membershipbased method that optimally represent data points in each granule. The triangular fuzzy set membership function is designed according to the method outlined in [22-24]. For each segment, in the interval $[a, b]$ the membership function is established as

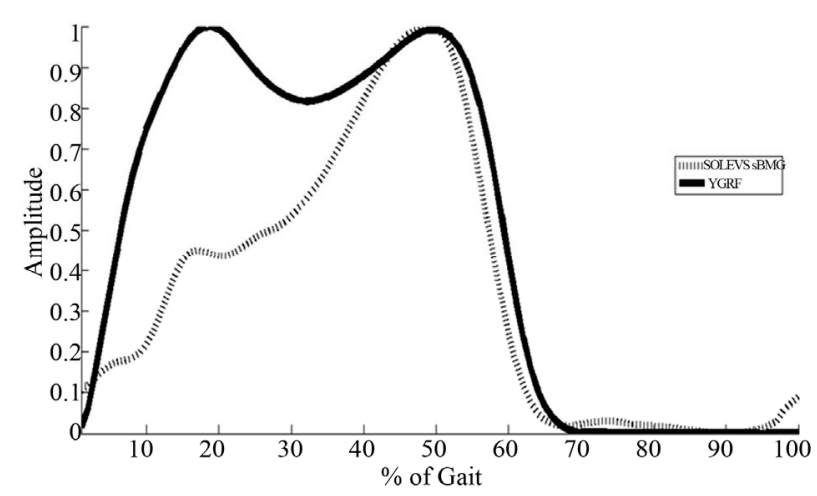

Figure 1. VGRF and soleus sEMG over one complete gait cycle. 


$$
\begin{aligned}
& \mu_{a, m, b}(x)=\frac{x-m}{m-a} \text { for } a \leq x \leq m \\
& \mu_{a, m, b}(x)=\frac{b-x}{b-m} \text { for } m \leq x \leq b
\end{aligned}
$$

where $a$ is the left bound, $m$ is the modal or core value, and $b$ is the right bound. The median of each segment is taken as a modal value [23]. To obtain the fuzzy parameters $a$ and $b$, Equation (3) is solved for each segment [23,24]. This equation provides the optimal value of $a$ and $b$ that satisfies the experimental significance and specificity requirement we need to have in each granule.

$$
Q(a, b)=\max \frac{\sum_{i=1}^{k} \mu_{a, m, b}\left(x_{i}\right)}{b-a}
$$

where $\mathrm{k}$ is the number of data points in each segment and $x_{i}$ is data point in the respective segment or phase. Figure 2 represents an example of Soleus sEMG signal granulated into seven phases.

\subsection{Granular Matrix and Calculation of Fuzzy Similarity}

We form the granular matrix, $G$ for each gait phase represented by $(a, m, b)$ [22], an example of such $3 \times 7$ matrix is shown in Equation (4).

$$
G=\left(\begin{array}{c|ccccccc} 
& P_{1} & P_{2} & P_{3} & P_{4} & P_{5} & P_{6} & P_{7} \\
\hline a & \cdot & \cdot & \cdot & . & \cdot & \cdot & \cdot \\
m & \cdot & . & \cdot & \cdot & \cdot & \cdot & \cdot \\
b & . & . & . & . & . & . & .
\end{array}\right)
$$

where $P_{i}(i=1, \cdots, 7)$ represent gait phases.

The fuzzy similarity (FS) [19] between two granulated

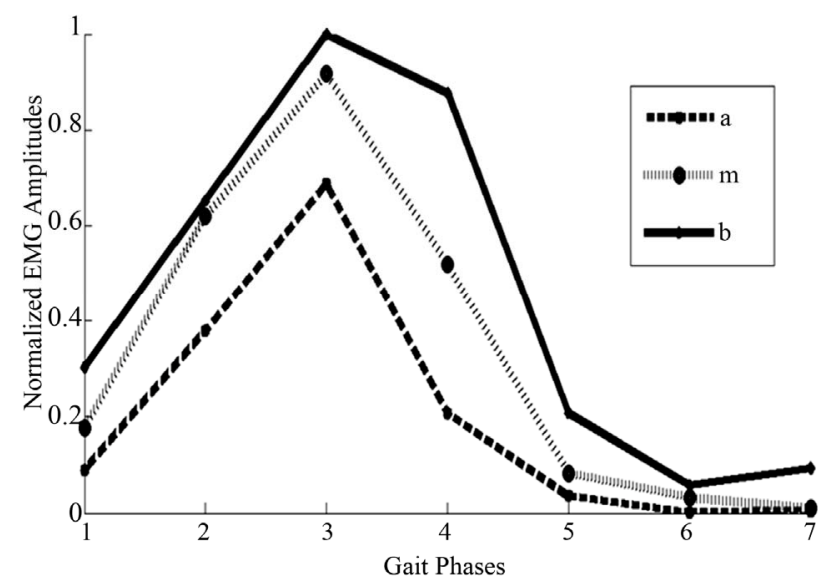

Figure 2. Granulated soleus sEMG represented through the fuzzy-parameters $a, m$, and $b$. gait variables represented by $G=g_{i j}$ and $H=h_{i j}$ can be calculated as

$$
G * H=\frac{\min \left(g_{i j}, h_{i j}\right)}{\max \left(g_{i j}, h_{i j}\right)}
$$

where the symbol “*” stands for the fuzzy-correlation operator, "min" for the fuzzy-intersection and "max" for fuzzy-union. In this study $G$ represent the granulated gait variable for reference group and $H$ could mean the respective granulated variable for a subject with MS or CP. And the FS defines the similarity between the reference and test subject in each gait phases. The FS is within a range between 0 and 1 . FS value of zero signifies no similarity at all and 1 represents 100\% similarity. An FS value closer to 1 indicates higher degree of similarity and FS values close to zero show little or no similarity. FS measure is chosen to make it consistent with the fuzzygranulation algorithm; otherwise any similarity measure that scales between 0 and 1 could as well be used.

\section{EXPERIMENTAL DESIGN AND METHODS}

\subsection{Participants}

The institutional review board (IRB) of The University of Texas at El Paso approved this study. Subjects obtained explanations about the study and are asked to sign informed consent prior to participation. Twenty-two male (Age: $24.5 \pm 4.0$ years; Weight: $74.6 \pm 14.6 \mathrm{~kg}$; Height: $173.0 \pm 8.4 \mathrm{~cm})$ and twelve female control able-bodied subjects (Age: $25.2 \pm 5.5$ years; Weight: $63.7 \pm$ $9.7 \mathrm{~kg}$; Height: $164 \pm 5.8 \mathrm{~cm}$ ) with no history of gait abnormalities are recruited from the El Paso community. Table 1 shows the six MS patients (2 female and 4 male), and four CP (1 female and 3 male) subjects selected for this study.

Table 1. Patient anthropometric data.

\begin{tabular}{cccccc}
\hline Patient & Gender & Age & Weight $(\mathrm{kg})$ & Height $(\mathrm{cm})$ & BMI \\
\hline${ }^{*}$ CP01 & Female & 26 & 44.9 & 151 & 26.6 \\
CP02 & Male & 17 & 69.5 & 162 & 26.5 \\
CP03 & Male & 17 & 68.3 & 164 & 25.4 \\
CP04 & Male & 55 & 88.9 & 172 & 30.1 \\
${ }^{*}$ MS01 & Female & 55 & 66.1 & 150 & 29.4 \\
MS02 & Female & 40 & 61.2 & 165 & 22.5 \\
MS03 & Male & 62 & 77.7 & 162 & 29.6 \\
MS04 & Male & 37 & 130.7 & 181 & 39.9 \\
MS05 & Male & 45 & 124.2 & 178 & 39.2 \\
MS06 & Male & 28 & 82.5 & 194.5 & 21.8 \\
\hline${ }^{*}$ CP = Cerebral palsy; ${ }^{*}$ MS $=$ Multiple sclerosis. & &
\end{tabular}




\subsection{Data Acquisition and Processing}

All subjects in this study able-bodied, MS and CP subjects, performed free barefoot treadmill walking at their comfortable speed for 180 seconds. The speed of the treadmill is controllable and can be set at the subject's comfortable speed. A dual-belt treadmill (Bertec, Corporations, USA) is used to measure the ground reaction forces (GRFs) in three-dimensions. The force plates measure the ground reaction forces at $100 \mathrm{~Hz}$ sampling frequency. VGRF was filtered using a second order Butterworth low pass filter with cut-off frequency of $20 \mathrm{~Hz}$ to remove the noise. VGRF component was used to define the gait cycles. To represent each cycle (stride) in percentage, time-normalization was done by re-sampling [25]. An average VGRF for one cycle was calculated from 100 strides. To allow inter-subject comparison, the VGRF was normalized by the weight in kilograms of respective subject. Male and female ablebodied subjects VGRF data were analyzed separately and averaged to establish a separate reference for male and female.

The dynamic sEMG data for four selected muscles for right side: soleus (SOL), tibialis anterior (TA), gas-trocnemius laterialis (LG), and vastus laterialis (VL), are measured by the Delsys Myomonitor wirless EMG system (Delsys Inc., Boston, MA, USA). The sEMG data acquisition was sampled at $1000 \mathrm{~Hz}$ and electrodes were placed according to [26]. All sEMG signal data were filtered by band pass 3rd order Butterworth filter with cutoff frequency between 20 and $250 \mathrm{~Hz}$ to remove low and high frequency noise. Re-sampling was done to convert into percentage of gait cycle and to make the length of each sEMG signal the same for each stride [27]. The average sEMG for each muscle was determined from 100 cycles sEMG data. The amplitudes of sEMG were normalized based on the maximum average to allow comparison between individuals. Male and female sEMG reference was then built separately by averaging the respective gender group sEMG. The reference VGRF and sEMG are divided into seven parts based on the percentage of each gait phases. The triangular fuzzy-membership function parameters $\mathrm{a}, \mathrm{m}$, and $\mathrm{b}$ were constructed for each segment using Equations (1) and (2). Each phase of a given gait cycle is now represented by the parameters $(a, m, b)$ and a $3 \times 7$ granular matrices that represents the full gait cycle. Reference granular matrixes that represent the able-bodied group are built for VGRF and the sEMG data. For each MS and CP patient similar data processing scheme is followed and representative granular matrix for each patient subject is constructed. The fuzzy similarity of the reference matrix and that of a patient (CP and MS in this case) is then determined by Equation (4).

\section{RESULTS AND DISCUSSION}

The proposed method is applied to establish similarity of VGRF and sEMG data of the reference able-bodied and patients group (CP and MS) in each gait phases. Each entry in the result table expresses the similarity of fuzzy parameters $(a, m, b)$ of patients and control group.

\subsection{Vertical Ground Reaction Force}

The fuzzy similarity of the of right and left VGRF in each gait phase for the $4 \mathrm{CP}$ and 6 MS subjects are presented in Tables 2 and 3. Different FS values are reported for left and right VGRF, which can help assess both legs separately. The values in the tables express how similar the patient fuzzy parameters $(a, m$, and $b)$ are with the corresponding reference values. A smaller FS values signifies deviation or variations from the expected behaviors. On the other hand a bigger FS values, imply sign of closeness to the normal patterns and functioning. We observe lower FS values in gait phase 5 (initial-swing), 6 (mid-swing), and 7 (terminal swing) for most of CP and MS patients. Relatively, most patient subjects have degraded FS values in phase 6. Phase 7 FS values are comparatively higher than phase 5 and 6 values. The fuzzy parameter, $b$ (right bound) has higher FS values compared with the corresponding fuzzy parameters a (left bound), and $\mathrm{m}$ (the core) in phases 5-7. Smaller FS values in phase 5-7 signify that most of patients have gait problems during swing phase.

\subsection{Muscle Activity}

The degree of FS for the right leg for four lower-extremity muscles of CP patients' and the able-bodied group is presented in Table 4.

Each CP patient has different FS values that depend on individual impairment level and intervention or therapy undergone. The FS values for the right-soleus muscle are smaller during $\mathrm{P}_{5}, \mathrm{P}_{6}$, and $\mathrm{P}_{7}$. Particularly $\mathrm{CP} 01$ and CP02 have lowered FS values for soleus in $\mathrm{P}_{5}$ and $\mathrm{P}_{6}$. CP04 has relatively higher FS values in the swing phase for soleus-muscle. The soleus muscle is expected to be activated at the start of the stance phase (loading-response) and attains its maximum during the final phase of the stance (pre-swing). Soleus remains relaxed during the swing phase. CP01 has noticeable, very low FS for soleus during the first three phases where this muscle is expected to achieve full activation. This is sign for abnormal or under normal activation of the respective muscle.

CP02 and CP03 have smaller FS values for $\mathrm{P}_{1}$ (loading-response), this may be an indication of delayed Soleus muscle activation. Tibialis anterior (TA) of CP01, $\mathrm{CP} 02$, and $\mathrm{CP0} 3$ have very small FS values in the first 
Table 2. FS values for VGRF for the CP patients.

\begin{tabular}{|c|c|c|c|c|c|c|c|c|}
\hline & & $\mathrm{P}_{1}$ & $\mathrm{P}_{2}$ & $\mathrm{P}_{3}$ & $\mathrm{P}_{4}$ & $\mathrm{P}_{5}$ & $\mathrm{P}_{6}$ & $\mathrm{P}_{7}$ \\
\hline \multirow{8}{*}{ СР01 } & \multicolumn{8}{|c|}{ Right VGRF } \\
\hline & $a$ & 0.884 & 0.679 & 0.987 & 0.904 & 0.800 & 0.272 & 0.778 \\
\hline & $m$ & 0.908 & 0.864 & 0.981 & 0.863 & 0.253 & 0.325 & 0.482 \\
\hline & $b$ & 0.022 & 0.951 & 0.982 & 0.877 & 0.900 & 0.704 & 0.737 \\
\hline & \multicolumn{8}{|c|}{ Left VGRF } \\
\hline & $a$ & 0.911 & 0.956 & 0.896 & 0.744 & 0.545 & 0.206 & 0.289 \\
\hline & $m$ & 0.745 & 0.946 & 0.988 & 0.895 & 0.138 & 0.133 & 0.927 \\
\hline & $b$ & 0.725 & 0.875 & 0.896 & 0.315 & 0.977 & 0.903 & 0.996 \\
\hline \multirow{8}{*}{ СР02- } & \multicolumn{8}{|c|}{ Right VGRF } \\
\hline & $a$ & 0.773 & 0.775 & 0.853 & 0.579 & 0.046 & 0.025 & 0.295 \\
\hline & $m$ & 0.651 & 0.936 & 0.937 & 0.995 & 0.057 & 0.002 & 0.357 \\
\hline & $b$ & 0.789 & 0.825 & 0.920 & 0.884 & 0.779 & 0.816 & 0.855 \\
\hline & \multicolumn{8}{|c|}{ Left VGRF } \\
\hline & $a$ & 0.719 & 0.671 & 0.872 & 0.824 & 0.082 & 0.010 & 0.088 \\
\hline & $m$ & 0.512 & 0.805 & 0.931 & 0.868 & 0.084 & 0.003 & 0.268 \\
\hline & $b$ & 0.536 & 0.960 & 0.820 & 0.826 & 0.726 & 0.537 & 0.538 \\
\hline \multirow{8}{*}{ СР03- } & \multicolumn{8}{|c|}{ Right VGRF } \\
\hline & $a$ & 0.501 & 0.688 & 0.939 & 0.538 & 0.064 & 0.006 & 0.358 \\
\hline & $m$ & 0.876 & 0.983 & 0.980 & 0.975 & 0.063 & 0.004 & 0.438 \\
\hline & $b$ & 0.119 & 0.897 & 0.862 & 0.543 & 0.803 & 0.290 & 0.703 \\
\hline & \multicolumn{8}{|c|}{ Left VGRF } \\
\hline & $a$ & 0.582 & 0.537 & 0.876 & 0.599 & 0.101 & 0.006 & 0.514 \\
\hline & $m$ & 0.833 & 0.859 & 0.975 & 0.985 & 0.079 & 0.005 & 0.397 \\
\hline & $b$ & 0.627 & 0.960 & 0.790 & 0.757 & 0.899 & 0.820 & 0.786 \\
\hline \multirow{8}{*}{ СР04 } & \multicolumn{8}{|c|}{ Right VGRF } \\
\hline & $a$ & 0.773 & 0.983 & 0.892 & 0.903 & 0.452 & 0.430 & 0.926 \\
\hline & $m$ & 0.739 & 0.978 & 0.995 & 0.843 & 0.226 & 0.791 & 0.488 \\
\hline & $b$ & 0.853 & 0.935 & 0.748 & 0.663 & 0.111 & 0.555 & 0.585 \\
\hline & \multicolumn{8}{|c|}{ Left VGRF } \\
\hline & $a$ & 0.523 & 0.735 & 0.851 & 0.618 & 0.286 & 0.519 & 0.534 \\
\hline & $m$ & 0.681 & 0.965 & 0.937 & 0.982 & 0.224 & 0.821 & 0.625 \\
\hline & $b$ & 0.249 & 0.816 & 0.927 & 0.933 & 0.845 & 0.284 & 0.333 \\
\hline
\end{tabular}

three phase of the cycle. TA muscle is activated in the first phase and stays relaxed until the first part of the swing-phase. The smaller FS values during the first phase are evidence of improper activation of the respective muscle. Generally smaller FS values are indication of unusual muscle activity and that need to be addressed in the treatment process. This kind of quantification of muscle activity within the seven gait phases provides an individual based assessment tool that can be tailored for treatment planning and interventions. Similar analysis can be done for the remaining two muscles from Table 4 for CP patients.
Table 3. FS values for VGRF for the MS patients.

\begin{tabular}{|c|c|c|c|c|c|c|c|c|}
\hline & & $\mathrm{P}_{1}$ & $\mathrm{P}_{2}$ & $\mathrm{P}_{3}$ & $\mathrm{P}_{4}$ & $\mathrm{P}_{5}$ & $\mathrm{P}_{6}$ & $\mathrm{P}_{7}$ \\
\hline \multirow{8}{*}{ MS0 } & \multicolumn{8}{|c|}{ Right VGRF } \\
\hline & $a$ & 0.940 & 0.941 & 0.799 & 0.460 & 0.538 & 0.327 & 0.096 \\
\hline & $m$ & 0.831 & 0.911 & 0.885 & 0.868 & 0.084 & 0.234 & 0.808 \\
\hline & $b$ & 0.632 & 0.826 & 0.897 & 0.995 & 0.998 & 0.356 & 0.360 \\
\hline & \multicolumn{8}{|c|}{ Left VGRF } \\
\hline & $a$ & 0.705 & 0.999 & 0.884 & 0.750 & 0.969 & 0.399 & 0.551 \\
\hline & $m$ & 0.871 & 0.904 & 0.917 & 0.733 & 0.830 & 0.499 & $0.46 \mathrm{~S}$ \\
\hline & $b$ & 0.925 & 0.951 & 0.952 & 0.900 & 0.841 & 0.710 & $0.70 s$ \\
\hline \multirow{8}{*}{ SO2 } & \multicolumn{8}{|c|}{ Right VGRF } \\
\hline & $a$ & 0.629 & 0.790 & 0.880 & 0.419 & 0.097 & 0.589 & 0.692 \\
\hline & $m$ & 0.950 & 0.984 & 0.939 & 0.847 & 0.055 & 0.278 & 0.864 \\
\hline & $b$ & 0.900 & 0.951 & 0.982 & 0.995 & 0.577 & 0.793 & 0.830 \\
\hline & \multicolumn{8}{|c|}{ Left VGRF } \\
\hline & $a$ & 0.746 & 0.767 & 0.873 & 0.938 & 0.514 & 0.579 & 0.973 \\
\hline & $m$ & 0.832 & 0.937 & 0.937 & 0.763 & 0.349 & 0.706 & 0.825 \\
\hline & $b$ & 0.583 & 0.951 & 0.952 & 0.995 & 0.920 & 0.932 & 0.937 \\
\hline \multirow{8}{*}{ IS03 } & \multicolumn{8}{|c|}{ Right VGRF } \\
\hline & $a$ & 0.098 & 0.580 & 0.870 & 0.537 & 0.027 & 0.002 & 0.995 \\
\hline & $m$ & 0.523 & 0.786 & 0.916 & 0.938 & 0.044 & 0.001 & 0.801 \\
\hline & $b$ & 0.746 & 0.960 & 0.960 & 0.817 & 0.820 & 0.868 & $0.18 \mathrm{~s}$ \\
\hline & \multicolumn{8}{|c|}{ Left VGRF } \\
\hline & $a$ & 0.185 & 0.584 & 0.938 & 0.464 & 0.027 & 0.005 & 0.100 \\
\hline & $m$ & 0.528 & 0.710 & 0.917 & 0.926 & 0.041 & 0.001 & 0.575 \\
\hline & $b$ & 0.811 & 0.725 & 0.948 & 0.933 & 0.552 & 0.603 & 0.444 \\
\hline \multirow{8}{*}{ MS04 } & \multicolumn{8}{|c|}{ Right VGRF } \\
\hline & $a$ & 0.681 & 0.768 & 0.849 & 0.471 & 0.044 & 0.004 & 0.753 \\
\hline & $m$ & 0.539 & 0.874 & 0.938 & 0.925 & 0.048 & 0.006 & 0.662 \\
\hline & $b$ & 0.563 & 0.960 & 0.744 & 0.933 & 0.565 & 0.891 & 0.503 \\
\hline & \multicolumn{8}{|c|}{ Left VGRF } \\
\hline & $a$ & 0.465 & 0.485 & 0.833 & 0.799 & 0.092 & 0.013 & 0.486 \\
\hline & $m$ & 0.504 & 0.925 & 0.908 & 0.831 & 0.086 & 0.019 & 0.488 \\
\hline & $b$ & 0.660 & 0.840 & 0.636 & 0.933 & 0.549 & 0.888 & 0.79 \\
\hline \multirow{8}{*}{ MS05 } & \multicolumn{8}{|c|}{ Right VGRF } \\
\hline & $a$ & 0.032 & 0.954 & 0.879 & 0.713 & 0.427 & 0.062 & 0.660 \\
\hline & $m$ & 0.996 & 0.947 & 0.952 & 0.982 & 0.362 & 0.887 & 0.615 \\
\hline & $b$ & 0.633 & 0.764 & 0.963 & 0.827 & 0.219 & 0.007 & 0.010 \\
\hline & \multicolumn{8}{|c|}{ Left VGRF } \\
\hline & $a$ & 0.348 & 0.982 & 0.850 & 0.882 & 0.717 & 0.403 & 0.958 \\
\hline & $m$ & 0.906 & 0.939 & 0.934 & 0.964 & 0.815 & 0.960 & 0.817 \\
\hline & $b$ & 0.015 & 0.933 & 0.927 & 0.811 & 0.350 & 0.238 & 0.240 \\
\hline \multirow{8}{*}{ MS06 } & \multicolumn{8}{|c|}{ Right VGRF } \\
\hline & $a$ & 0.949 & 0.596 & 0.833 & 0.621 & 0.469 & 0.128 & 0.194 \\
\hline & $m$ & 0.571 & 0.937 & 0.915 & 0.998 & 0.102 & 0.133 & 0.411 \\
\hline & $b$ & 0.939 & 0.943 & 0.858 & 0.760 & 0.917 & 0.781 & 0.733 \\
\hline & \multicolumn{8}{|c|}{ Left VGRF } \\
\hline & $a$ & 0.758 & 0.504 & 0.913 & 0.504 & 0.212 & 0.030 & 0.342 \\
\hline & $m$ & 0.616 & 0.995 & 0.963 & 0.937 & 0.074 & 0.033 & 0.701 \\
\hline & $b$ & 0.776 & 0.960 & 0.707 & 0.705 & 0.853 & 0.814 & 0.840 \\
\hline
\end{tabular}


Table 4. FS values for four muscles of CP patients.

\begin{tabular}{|c|c|c|c|c|c|c|c|c|}
\hline & & $\mathrm{P}_{1}$ & $\mathrm{P}_{2}$ & $\mathrm{P}_{3}$ & $\mathrm{P}_{4}$ & $\mathrm{P}_{5}$ & $\mathrm{P}_{6}$ & $\mathrm{P}_{7}$ \\
\hline \multirow{16}{*}{ CP01 } & \multicolumn{8}{|c|}{ RSOL } \\
\hline & $a$ & 0.263 & 0.054 & 0.068 & 0.894 & 0.131 & 0.006 & 0.022 \\
\hline & $m$ & 0.297 & 0.108 & 0.131 & 0.600 & 0.101 & 0.092 & 0.040 \\
\hline & $b$ & 0.379 & 0.642 & 0.232 & 0.983 & 0.127 & 0.136 & 0.190 \\
\hline & \multicolumn{8}{|c|}{ RTA } \\
\hline & $a$ & 0.012 & 0.631 & 0.012 & 0.011 & 0.058 & 0.046 & 0.025 \\
\hline & $m$ & 0.049 & 0.228 & 0.076 & 0.031 & 0.322 & 0.028 & 0.065 \\
\hline & $b$ & 0.143 & 0.429 & 0.123 & 0.077 & 0.228 & 0.061 & 0.034 \\
\hline & \multicolumn{8}{|c|}{ RLG } \\
\hline & $a$ & 0.121 & 0.889 & 0.024 & 0.132 & 0.015 & 0.004 & 0.010 \\
\hline & $m$ & 0.165 & 0.727 & 0.145 & 0.825 & 0.025 & 0.033 & 0.030 \\
\hline & $b$ & 0.228 & 0.559 & 0.332 & 0.704 & 0.049 & 0.049 & 0.094 \\
\hline & \multicolumn{8}{|c|}{ RVL } \\
\hline & $a$ & 0.728 & 0.779 & 0.156 & 0.025 & 0.005 & 0.002 & 0.178 \\
\hline & $m$ & 0.624 & 0.675 & 0.201 & 0.040 & 0.005 & 0.009 & 0.804 \\
\hline & $b$ & 0.544 & 0.453 & 0.430 & 0.438 & 0.244 & 0.065 & 0.350 \\
\hline \multirow{16}{*}{$\mathrm{CP} 02$} & \multicolumn{8}{|c|}{ RSOL } \\
\hline & $a$ & 0.340 & 0.704 & 0.952 & 0.394 & 0.088 & 0.035 & 0.059 \\
\hline & $m$ & 0.391 & 0.950 & 0.793 & 0.707 & 0.176 & 0.176 & 0.080 \\
\hline & $b$ & 0.342 & 0.866 & 0.791 & 0.907 & 0.384 & 0.168 & 0.601 \\
\hline & \multicolumn{8}{|c|}{ RTA } \\
\hline & $a$ & 0.039 & 0.385 & 0.362 & 0.074 & 0.232 & 0.011 & 0.977 \\
\hline & $m$ & 0.042 & 0.401 & 0.925 & 0.184 & 0.079 & 0.032 & 0.866 \\
\hline & $b$ & 0.184 & 0.176 & 0.851 & 0.606 & 0.051 & 0.481 & 0.828 \\
\hline & \multicolumn{8}{|c|}{ RLG } \\
\hline & $a$ & 0.087 & 0.552 & 0.414 & 0.576 & 0.085 & 0.009 & 0.044 \\
\hline & $m$ & 0.373 & 0.540 & 0.515 & 0.730 & 0.175 & 0.121 & 0.049 \\
\hline & $b$ & 0.303 & 0.642 & 0.644 & 0.579 & 0.415 & 0.108 & 0.217 \\
\hline & \multicolumn{8}{|c|}{ RVL } \\
\hline & $a$ & 0.089 & 0.078 & 0.018 & 0.005 & 0.003 & 0.046 & 0.466 \\
\hline & $m$ & 0.335 & 0.490 & 0.074 & 0.011 & 0.009 & 0.045 & 0.101 \\
\hline & $b$ & 0.476 & 0.150 & 0.354 & 0.014 & 0.996 & 0.114 & 0.235 \\
\hline \multirow{16}{*}{ CP03- } & \multicolumn{8}{|c|}{ RSOL } \\
\hline & $a$ & 0.277 & 0.658 & 0.930 & 0.315 & 0.098 & 0.088 & 0.979 \\
\hline & $m$ & 0.322 & 0.779 & 0.877 & 0.724 & 0.121 & 0.758 & 0.581 \\
\hline & $b$ & 0.334 & 0.652 & 0.879 & 0.918 & 0.258 & 0.211 & 0.739 \\
\hline & \multicolumn{8}{|c|}{ RTA } \\
\hline & $\mathrm{a}$ & 0.022 & 0.283 & 0.328 & 0.075 & 0.325 & 0.085 & 0.706 \\
\hline & $\mathrm{m}$ & 0.090 & 0.302 & 0.969 & 0.205 & 0.113 & 0.151 & 0.960 \\
\hline & $\mathrm{b}$ & 0.152 & 0.200 & 0.717 & 0.589 & 0.073 & 0.771 & 0.828 \\
\hline & \multicolumn{8}{|c|}{ RLG } \\
\hline & $a$ & 0.872 & 0.511 & 0.542 & 0.551 & 0.050 & 0.233 & 0.543 \\
\hline & $m$ & 0.435 & 0.389 & 0.569 & 0.673 & 0.096 & 0.065 & 0.209 \\
\hline & $b$ & 0.458 & 0.932 & 1.000 & 0.970 & 0.218 & 0.057 & 0.845 \\
\hline & \multicolumn{8}{|c|}{ RVL } \\
\hline & $a$ & 0.148 & 0.152 & 0.054 & 0.046 & 0.012 & 0.001 & 0.247 \\
\hline & $m$ & 0.734 & 0.785 & 0.225 & 0.075 & 0.017 & 0.003 & 0.880 \\
\hline & $b$ & 0.771 & 0.577 & 1.000 & 1.000 & 0.282 & 0.087 & 0.865 \\
\hline
\end{tabular}

\section{Continued}

\begin{tabular}{cccccccc}
\hline \multicolumn{8}{c}{ RSOL } \\
\hline$a$ & 0.888 & 0.952 & 0.935 & 0.634 & 0.686 & 0.206 & 0.054 \\
$m$ & 0.621 & 0.916 & 0.930 & 0.458 & 0.728 & 0.761 & 0.052 \\
$b$ & 0.477 & 0.890 & 1.000 & 0.491 & 0.893 & 0.979 & 0.312 \\
\hline \multicolumn{7}{c}{ RTA } \\
\hline$a$ & 0.697 & 0.373 & 0.205 & 0.003 & 0.102 & 0.644 & 0.522 \\
$m$ & 0.603 & 0.204 & 0.267 & 0.008 & 0.394 & 0.785 & 0.525 \\
$\mathrm{CP} 04$ & 0.764 & 0.410 & 0.138 & 0.080 & 0.633 & 0.820 & 0.978 \\
\hline & & \multicolumn{7}{c}{$\mathrm{RLG}$} \\
\hline$a$ & 0.173 & 0.161 & 0.854 & 0.261 & 0.318 & 0.074 & 0.121 \\
$m$ & 0.318 & 0.949 & 0.968 & 0.650 & 0.161 & 0.520 & 0.171 \\
$b$ & 0.503 & 0.733 & 1.000 & 0.918 & 0.232 & 0.352 & 0.829 \\
\hline & & \multicolumn{7}{c}{ RVL } \\
\hline$a$ & 0.476 & 0.263 & 0.090 & 0.057 & 0.023 & 0.029 & 0.653 \\
$m$ & 0.605 & 0.561 & 0.256 & 0.119 & 0.049 & 0.077 & 0.913 \\
$b$ & 0.775 & 0.974 & 1.000 & 1.000 & 0.282 & 0.420 & 0.393 \\
\hline
\end{tabular}

The comparison of muscle activity of MS patients and able-bodied group is displayed in Table 5. Again, in the case of MS subjects, wide ranges of FS values are observed. This variation is due to individual difference in gait deficit and the level of MS disease progression. FS values for MS01 and MS03 in swing phase $\left(\mathrm{P}_{5}, \mathrm{P}_{6}\right.$, and $\mathrm{P}_{7}$ ) is relatively lower than the other MS subjects for the four muscles. MS02 has shown relatively improved FS values in most of the seven gait phases. We present muscle activity of the right leg, however the same analysis could also be done on the left leg.

The objective of this study is to investigate the possible application of granular computing to quantify gait parameters within the seven gait phases. The result of this study has shown to be effective in providing individual specific information about impairment level of CP and MS patients in comparison with age and sex matched able-bodied group. Analysis of VGRF revealed most of $\mathrm{CP}$ and MS subjects have smaller degree of similarity during the swing $\left(\mathrm{P}_{5}, \mathrm{P}_{6}\right.$, and $\left.\mathrm{P}_{7}\right)$ phase. This is the part of the gait cycle where the respective leg is in the air to switch to the other leg. A smaller FS value at this part of the gait cycle signifies subject's difficulty to alternatively switch between the right and the left leg that may lead to gait instability and reduced balance. This result is in agreement with previous studies $[3,27,28]$, where reducespeed, and impaired balance has been reported even in early MS subjects. On the other hand smaller FS values may also imply abnormal or altered patterns in the patients' VGRF signal that could change the frequency content of the signal. Frequency content analysis of VGRF of MS patients has shown significantly lower than able-bodied group [6]. 
Table 5. FS values for four muscles of MS patients.

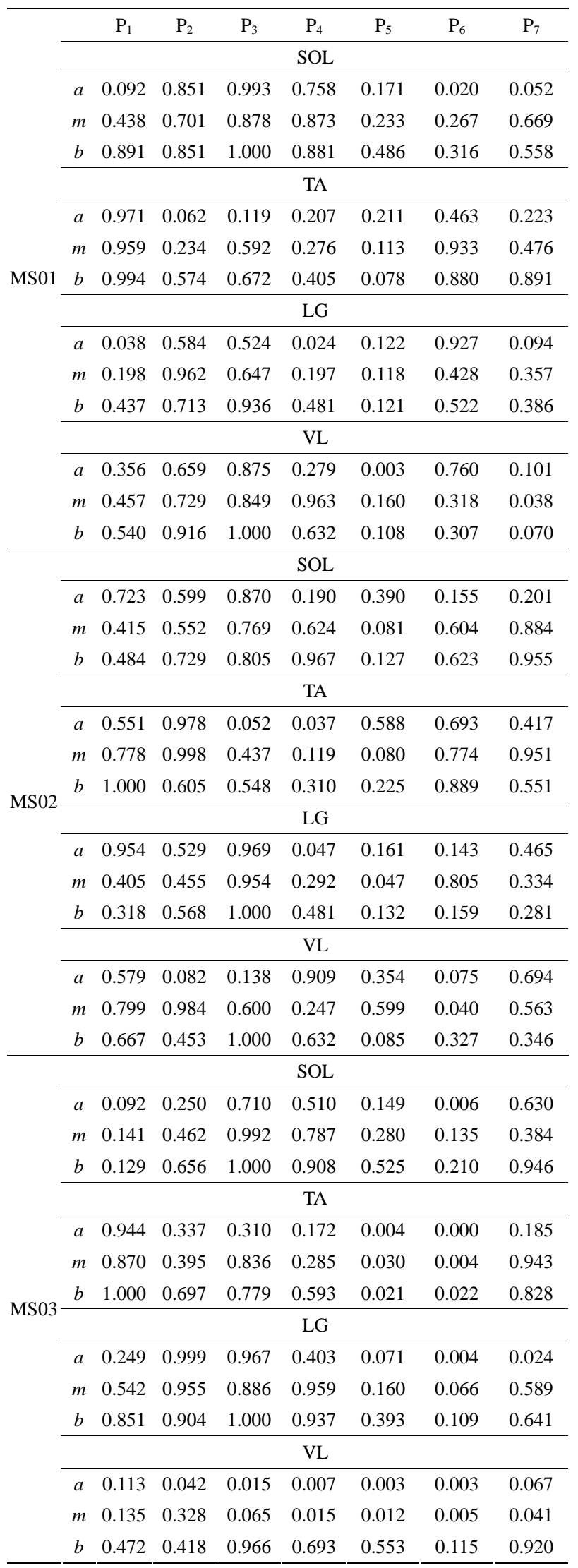

Continued

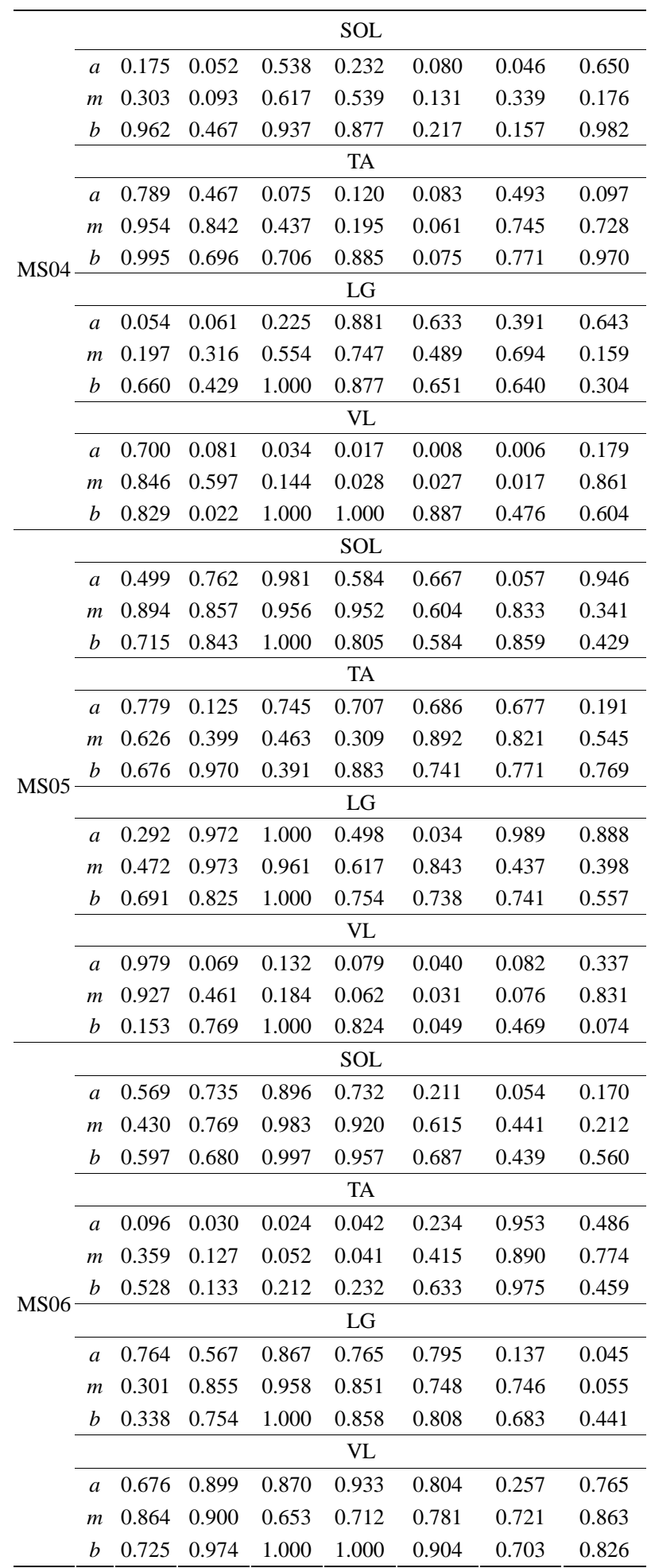

FS values for muscle activities of the four-selected muscle display a broad range of values due to difference between subjects. However, one can infer important individual information from the given FS values. Smaller FS values are symptoms of irregularities and deviation from 
the expected normal activity. Since, each CP and MS subject is different in the type of gait deficit and the level of impairment, the calculated FS values for one muscle may not follow the same trend in all subjects. For example, lower FS values in phases 5-7 are observed, for most CP subjects, however CP04 has relatively higher FS values in the swing phase. This process of measuring similarity within the seven gait phases, furnishes individual centered information that can relate more to the individual. Further, since the information is available for each phase, it is easier to identify specific problem within gait phases. Identifying specific problems at a particular part of the walking process helps to single out the type of treatment and rehabilitation procedure needed.

One possible limitation to this study is that FS values could be affected by the speed of walking on the treadmill, because subjects walk at different speed over ground and treadmill. However, an effort has been made to minimize this effect and allow inter-subject comparison by normalizing the data using Equation (1) before granulation. Further, the VGRF data are normalized by the body weight, and sEMG data are normalized by the maximum value to permit comparisons between subjects. In this research, the authors employed an automatic detection of the gait cycles using VGRF values. A heelstrike was defined at the point where the VGRF records a value above a threshold based on the noise-level of the signal. A toe-off is marked when VGRF drops below the threshold. A complete gait cycle then covers the whole period from the initial rise of VGRF to the next above threshold value. This is very robust method compared with other automatic gait cycle detection algorithms based on inertial sensors [29]. Because, inertial sensors, like accelerometers, usually are very sensitive and the reading highly depends on the orientation and have an associated offsets.

The proposed method is not a diagnostic tool like the one presented in [30,31]; rather it is an assessment or evaluation model. It provides valuable information regarding the relative impairment level compared to healthy control group. It can't be used to detect the presence of CP or MS or classify CP and MS. This approach can be extended for assessment of gait related problems due to other neurological disorder as well.

\section{CONCLUSION}

We apply fuzzy-granular computing to quantify VGRF and sEMG values within the seven gait phases for the purpose of assessing and identifying specific gait deficits associated with CP and MS subjects. The proposed approach is shown to be effective in providing individual based assessment information for specific part of the gait cycle. We demonstrated the possible use of fuzzy similarity measure values between age and sex matched con- trol able-bodied group and patient group (MS and CP) as a way of quantifying assessment of impairment level or identifying associated gait deficit. This approach not only enables to evaluate very specific gait defects but also pinpoints where the problem is within the gait cycle. Identification of abnormalities at specific point provides valuable information on the kind of treatment or intervention that can be prescribed. This individual based gait assessment information, can be integrated in clinical setting and provide crucial knowledge for individual follow up and rehabilitation process.

\section{ACKNOWLEDGEMENTS}

The authors would like to acknowledge the Computational Science Program of University of Texas at El Paso and the Stern foundation for their financial support. These funds were used to support graduate students in this project. The authors would like to thank the stern foundation for their financial support for our lab facility. The authors also would like to thank all subjects who participated in this study.

\section{REFERENCES}

[1] Whittle, M.W. (2007) Gait analysis an introduction. 4th Edition, Butterworth-Heineman, Elsevier, Amsterdam.

[2] Shakespeare, D., Boggild, M. and Young, C.A. (2003) Anti-spasticity agents for multiple sclerosis. Cochrane Database of Systematic Reviews, 4.

[3] Martin, C.L., Philips, B.A., Kilpatrick, T.J., Butzkueven, H., Tubridy, N., MacDonald, E. and Galea, M.P. (2006) Gait and balance impairment in early multiple sclerosis in the absence of clinical disability. Multiple Sclerosis, 12 , 620-628. doi:10.1177/1352458506070658

[4] Sosnoff, J.J., Sandroff, B.M. and Motl, R.W. (2012) Quantifying gait abnormalities in persons with multiple sclerosis with minimal disability. Gait \& Posture, 36, 154-156. doi:10.1016/j.gaitpost.2011.11.027

[5] Crenshaw, S.J., Royer, T.D., Richards, J.G. and Huson, D. J. (2006) Gait variability in people with multiple sclerosis. Multiple Sclerosis, 12, 613-619. doi:10.1177/1352458505070609

[6] Wurdeman, S.R., Huisinga, J.M., Filipi, M. and Stergiou, N. (2011) Multiple sclerios affects the frequency content in the vertical ground reaction forces during walking. Clinical Biomechanics, 26, 207-212. doi:10.1016/j.clinbiomech.2010.09.021

[7] Lee, E.H., Goh, J.C. and Bose, K. (1992) Value of gait analysis in the assessment of surgery in cerebral palsy. Archive of Physical Medicine and Rehabilitation, 73, 642-646.

[8] DeLuca, P.A., Davis, R.B., Ounpup, S. and Rose, S.R. (1997) Alterations in surgical decision making in patient with cerebral palsy based on three-dimensional gait analysis. Journal of Pediatric Orthopaedics, 17, 608-614.

[9] Filho, M.C., Yoshida, R., Carvalho, W., Stein, H.E. and Nova, N.F. (2008) Are recommendations from three-dimensional gait analysis associated with better postopera- 
tive outcomes in patients with cerebral palsy. Gait \& Posture, 28, 316-322.

doi:10.1016/j.gaitpost.2008.01.013

[10] Kawamura, C.M., Filho, M.C., Barreto, M.M., Asa, S.K., Juliano, Y. and Novo, N.F. (2007) Comparison between visual and three-dimensional gait analysis in patients with spastic diplegic cerebral palsy. Gait \& Posture, 25, 18-24. doi:10.1016/j.gaitpost.2005.12.005

[11] Chang, F.M., Hodes, J.T., Flynn, K.M. and Carollo, J.J. (2010) The role of gait analysis in treating gait abnormalities in cerebral palsy. Orthopedic Clinics of North America, 41, 489-506. doi:10.1016/j.ocl.2010.06.009

[12] Carriero, A., Zavatsky, A., Stebbins, J., Theologis, T. and Shefelbine, S.J. (2009) Determination of gait patterns in children with spastic diplegic cerebral palsy using principal components. Gait \& Posture, 29, 71-75. doi:10.1016/j.gaitpost.2008.06.011

[13] Bohm, H. and Doderlein, L. (2012) Gait asymmetries in children with cerebral palsy: Do they deteriorate with running. Gait \& Posture, 35, 322-327. doi:10.1016/j.gaitpost.2011.10.003

[14] Lauer, R.T., Stackhouse, C., Shewokis, P.A. and Smith, B.T. (2005) Assessment of wavelet analysis of gait in children with typical development and cerebral palsy. Journal of Biomechanics, 38, 1351-1357. doi:10.1016/j.jbiomech.2004.07.002

[15] Gestel, L.V., Laet, T.D., Lello, E.D., Bruyninckx, H., Molenaers, G., Van Campenhout, A., Aertbeliën, E., Schwartz, M., Wambacq, H., De Cock, P. and Desloovere, K. (2011) Probabilistic gait classification in children with cerebral palsy. A Bayesian Approach Research in Developmental Disabilities, 32, 2542-2552.

[16] Toro, B., Nester, C.J. and Farren, P.C. (2007) Cluster analysis for the extraction of Sagital gait patterns in children with cerebral palsy. Gait and Posture, 25, 157-165. doi:10.1016/j.gaitpost.2006.02.004

[17] O’Malley, M.J., Abel, M. and Damiano, D. (1997) Fuzzy clustering of children with cerebral palsy based on temporal-distance gait parameters. IEEE Transactions on Rehabilitation Engineering, 5, 300-309. doi:10.1109/86.650282

[18] Granata, K.P., Padua, D.A. and Abeel, M.F. (2004) Repeatability of surface EMG during gait in children. Gait \& Posture, 22, 346-350. doi:10.1016/j.gaitpost.2004.11.014

[19] Yu, H., Alaqtash, M., Spier, E. and Sarkodie-Gyan, T. (2010) Analysis of muscle activity during gait cycle using fuzzy-ruled based reasoning. Measurement, 43, 1106-1114. doi:10.1016/j.measurement.2010.04.010

[20] Alaqtash, M., Yu, H., Brower, R., Abdelgawad, A. and Sarkodie-Gyan, T. (2011) Application of wearable sensors for human gait analysis using fuzzy computational algorithm. Engineering Application of Artfical Intelligence, 24, 1018-1025.

doi:10.1016/j.engappai.2011.04.010

[21] Sarkodie-Gyan, T., Yu, H., Alaqtash, M., Abdelgawad, A., Spier, E. and Brower, R. (2011) Measurement of functional impairments in human locomotion using pattern analysis. Measurement, 44, 181-191. doi:10.1016/j.measurement.2010.09.043

[22] Yu, F., Chen, F. and Dong, K. (2005) A Granulationbased method for finding similarity between time series, granular computing. IEEE International Conference, 2527 July 2005, 700-703.

[23] Yu, F. and Pedrycz, W. (2009) The design of fuzzy information granules: Tradeoffs between specificity and experimental evidence. Applied Soft Computing, 9, 264 273. doi:10.1016/j.asoc.2007.10.026

[24] Peddrycz, W. and Gacek, A. (2002) Temporal granulations and its application to signal analysis. Information Science, 143, 47-71. doi:10.1016/S0020-0255(02)00179-2

[25] Winter, D.A. (2009) Biomechanics and motor control of human movement. 4th Edition, John Wiley \& Sons, Hoboken. doi:10.1002/9780470549148

[26] Cram, J.R., Kasman, G.S. and Holtz, J. (1998) Introduction to surface electromyography. Aspen Publishers, Maryland.

[27] De Stefano, A., Burridge, J.H., Yule, V.T. and Allen, R. (2003) Effect of gait cycle selection on EMG analysis during walking in adults and children with gait pathology. Gait \& Posture, 20, 92-1001. doi:10.1016/S0966-6362(03)00099-7

[28] Benedetti, M.G., Piperno, R., Simoncini, L., Bonato, P., Tonini, A. and Giannini, S. (1999) Gait abnormalities in minimal impaired multiple sclerosis patients. Multiple Sclerosis, 5, 363-368.

[29] Lopez-Meyer, P., Fulk, G.D. and Sazonov, E.S. (2011) Automatic detection of temporal gait parameters in postroke individuals. IEEE Transactions on Information Technology in Biomedicine, 15, 594-601. doi:10.1109/TITB.2011.2112773

[30] Tang, W., Tasch, W., Neerchal, N.K., Zahu, L. and Yarowsky, P. (2009) Measuring early pre-symptomatic changes in locomotion of SOD1-G93A rats-A rodent model of amyotrophic lateral sclerosis. Journal of Neuroscience Methods, 176, 254-262. doi:10.1016/j.jneumeth.2008.08.032

[31] Tang, W., McDowell, K., Limsam, M., Neerchal, N.K., Yarowsky, P. and Tasch, U. (2010) Locomotion analysis of Sprague-Dawley rats before and after injection 6OHDA. Behavioral Brain Research, 210, 131-133. 